\title{
IMPACTO DEL SISTEMA DE ANALISIS DE PELIGROS Y PUNTOS CRITICOS DE CONTROL EN LA DISMINUCIÓN DE LA CONTAMINACIÓN ALIMENTARIA Y GASTROENTERITIS EN UN HOSPITAL DE AYACUCHO, PERÚ
}

\author{
Gualberto Segovia $_{1}^{1, a}$, Nancy Santos $_{2, d}^{1, c}, J_{4}$ Julia Palomino ${ }^{1, c}$, Gutiérrez $E^{1}$, \\ Casavilca $R^{1}$, Vanessa García ${ }^{2, d}$, Vargas $E^{4}$, Jesus Paniagua ${ }^{3, b}$. Ketty Galvan ${ }^{2, c}$
}

1. Unidad de Epidemiología. Hospital Regional de Ayacucho.

2. Área Microbiología de Alimentos. Laboratorio de Referencia Regional Ayacucho.

3. Facultad de Industria Alimentaria. Universidad Nacional San Cristóbal de Huamanga. Ayacucho

a. Médico de Familia. b. Ingeniero en industrias alimentarias, c. Licenciada en enfermería. d. Bióloga

\section{RESUMEN}

Objetivo: Determinar el impacto del Sistema HACCP para el control de la contaminación de los alimentos y de las gastroenteritis intrahospitalarias. Material y métodos: Se trazó una línea de base a través de: 1) Inspección higiénico sanitaria del servicio de nutrición y dietética. 2) Estudio microbiológico de los alimentos proporcionados a los pacientes. 3) Estudio de prevalencia de las gastroenteritis intrahospitalarias; luego se intervino en el servicio con la implementación del Sistema HACCP, siguiendo los principios establecidos. Se realizó una vigilancia epidemiológica activa y selectiva sobre las gastroenteritis intrahospitalarias y un seguimiento de los alimentos proporcionados a los pacientes a través de estudios microbiológicos trimestrales por muestreo aleatorio estratificado por servicios. Resultados: En la línea de base las condiciones higiénico sanitaria del servicio fueron regulares, al final del estudio los resultados fueron muy buenos. El análisis microbiológicos de los alimentos al inicio reportaron contaminación en el $50 \%$ de los alimentos muestreados, al final de la intervención no se reportaron microorganismo alguno en las comidas servidas a los pacientes. En las Gastroenteritis Intrahospitalarias el estudio inicial reporto una prevalencia de $11,7 \%$, en el transcurso de la implementación del sistema HACCP las incidencias mensuales muestran una tendencia decreciente hasta $0,23 \%$. Conclusiones: EI Sistema HACCP contribuye de manera eficaz al control de la contaminación microbiológica de los alimentos hospitalarios y está relacionada directamente al control de las Gastroenteritis intrahospitalarias, ameritando su implementación en los servicios de nutrición de los hospitales. (Rev. méd. panacea 2011; 1:78 - 84).

Palabras clave: Vigilancia Epidemiológica, Agentes de Control de Microorganismos, Ingestión de Alimentos.(fuente: DeCS BIREME)

\section{IMPACT OF THE SYSTEM OF HAZARD ANALY SIS AND CRITICAL CONTROL POINTS FOR THE CONTROL OF THE GASTROENTERITIS AND THE MICROBIOLOGICAL POLLUTION OF THE FOOD IN A HOSPITAL. AYACUCHO, PERU}

\begin{abstract}
Objetive. Implementation of the HACCP System for the control of the microbiological pollution of food and nosocomial gastroenteritis. Materials and methods: a base line was planned across: 1) Inspection hygienic sanitary of the service of nutrition and dietetics. 2) Microbiological Study of the food provided to the patients. 3) Study of prevalence of nosocomial gastroenteritis; Then one intervened in the service with the implementation of the HACCP System, following the established beginning. There was realized an epidemiological active and selective vigilance on the nosocomial gastroenteritis and a followup of the food provided to the patients across microbiological quarterly studies by random sampling stratified by services. Results: In the base line one determined that the conditions hygienic sanitary of the service were regular at the end of the study the results were Very good. The microbiological analysis of the food to the beginning they brought pollution in $50 \%$ of the food sampled, at the end of the intervention the results of the food did not bring any microorganism in meals served to patients. In the nosocomial Gastroenteritis, the initial prevalence study brings a rate of $11.7 \%$, in the course of the implementation of the HACCP system, the monthly incidence rates show a downward trend from $14.4 \%$ to $0.23 \%$. Conclusions: The System HACCP contributes in an effective way to the control of the microbiological pollution of the hospital food and, is related directly to the control of the nosocomial Gastroenteritis. Therefore, he deserves his implementation in the services of nutrition of the hospitals. (Rev. méd. panacea 2011; 1:78-84).
\end{abstract}

Key words: Epidemiological Surveillance, Control Agents for Microorganisms, Eating. (Source: Mesh, NLM) 


\section{INTRODUCCIÓN}

En el último decenio se viene difundiendo y aplicando en la supervisión sanitaria de la producción y comercialización de alimentos, el sistema conocido como Análisis de Riesgos y Control de Puntos Críticos (HACCP), convirtiéndose en un eficaz instrumento para la obtención de alimentos inocuos para el consumo $(1,2)$.

El sistema HACCP busca ejercer el control a lo largo de todo el proceso identificando aquellas etapas en donde el producto está más expuesto a la contaminación, surgiendo como alternativa al sistema tradicional basado en el análisis del producto final, el cual requiere un muestreo tedioso y prohibitivo para asegurar el $100 \%$ de seguridad en los lotes de alimentos (3).

El HACCP es un método sistemático y continuo de prevención y organización, basado en fundamentos científicos, con el fin de lograr la seguridad de los alimentos, mejorar su calidad y disminuir las pérdidas ocasionadas por su alteración. Este sistema permite identificar peligros específicos y dar medidas para su control, garantizando así la inocuidad de alimentos en protección de consumidores y la salud pública $(4,5)$.

La empresa Pillsbury implementa este sistema por primera vez en los años 70 (2), como respuesta a la demanda de la NASA (Administración Nacional Espacial y Aeronáutica), que requería alimentos seguros en el $100 \%$, de manera que no puedan causar enfermedades a los astronautas.

A partir de 1973, la Food and Drug Administration (FDA) de los Estados Unidos de América dispone la implementación obligatoria del sistema en la industria de conservas enlatadas de baja acidez, haciéndolo luego extensivo a los productos pesqueros y a los productos cárnicos en 1995 y 1997 respectivamente. Desde inicios de los 90, Estados Unidos, Canadá y la Unión Europea vienen aplicando los principios del HACCP en sus directivas que regulan las reglas de higiene para la producción y comercialización de los alimentos $(6,7)$.

El Perú inicia la aplicación de este sistema HACCP en la industria de productos hidrobiológicos de exportación a partir de 1996. En 1997 se considera su aplicación en los programas de higiene de las empresas de alimentos para el proceso de autorización sanitaria (D.S. N01-97-SA). Es así que en 1998 se publica el Proyecto de Reglamentación sobre Vigilancia y Control considerándose la aplicación del HACCP en el control de calidad sanitaria e inocuidad de los productos de la industria de alimentos $(8,9)$, estableciéndose un plazo para su adopción (DIGESA, 1998).

Este sistema se ha generalizado en la industria alimentaria en nuestro país siendo su aplicación obligatoria sobre todo en alimentos hidrobiológicos destinados al comercio internacional. No ocurre lo mismo en el caso de alimentación institucional, en donde a excepción de pocos restaurantes turísticos y cadena de hoteles, este sistema no se aplica ni se conoce.

Se ha demostrado el importante rol que desempeñan los alimentos en la transmisión de enfermedades diarreicas agudas y el cólera. Estas enfermedades transmitidas por alimentos representan un problema serio para los hospitales (9), a pesar del subregistro existente por la poca vigilancia en nuestro país.

La investigación tuvo por objetivo determinar el impacto del sistema HACCP en el Hospital Regional de Ayacucho, tanto en el control de la contaminación de alimentos proporcionados por el servicio de nutrición como en las enfermedades transmitidas por estos alimentos a los pacientes.

\section{MATERIAL Y MÉTODOS}

En esta cohorte única realizada en el Hospital Regional de Ayacucho durante los meses de octubre del 2001 a diciembre del 2002, se evaluó la eficacia de una intervención (implementación del sistema HACCP) en el servicio de Nutrición y Dietética, para disminuir las enfermedades producidas por alimentos proporcionados por este servicio a los pacientes hospitalizados.

Con la finalidad de cumplir con los pre-requisitos exigidos para implementación del sistema HACCP se realizaron actividades iniciales relacionadas con el control de las condiciones ambientales, procedimientos y prácticas con la manipulación de los alimentos del personal dentro del servicio de Nutrición, que permita garantizar la seguridad de los alimentos producidos. Es así, que en el primer trimestre de la intervención se efectuaba la remodelación del ambiente, renovación e implementación del servicio de Nutrición con el apoyo del proyecto alemán GTZ, se realizó un estudio basal de la Situación Higiénico Sanitaria y Buenas Prácticas de Manufactura de este servicio usando el instrumento de inspección higiénico sanitario según el reglamento sobre vigilancia y control sanitario de alimentos y bebidas DS-007/98.S.A (10).

La calificación usada para la inspección higiénico sanitaria fue: Deficiente de 0 a 54 puntos; Regulares de 55 a 65 puntos; Buenas de 66 a 79 puntos; Muy buenas de 80 a 94 puntos y Excelente de 95 a 100 puntos.

Se intervino con un plan de capacitación teórico práctico consistente en Buenas Prácticas de Higiene (BPH), Procedimientos de Limpieza y Desinfección, Educación Sanitaria de Manipulación de alimentos, Análisis de Peligros, Determinación de Puntos Críticos de Control y de 
Límites Críticos, Vigilancia de Puntos Críticos, Medidas Correctivas, Verificación del Sistema HACCP y Registros del Sistema HACCP. La estrategia de las dos capacitación fue dialogo - expositiva y demostrativa in situ, con clases prácticas y ayudas audiovisuales; entregándose a cada participante al final de éstas manuales de autoaprendizaje, durante la implementación del sistema HACCP hubo un acompañamiento permanente por los investigadores, para medir la situación del servicio de nutrición y los conocimientos impartidos se efectuaron tres inspecciones higiénicos sanitarias al inicio de la intervención, al semestre y al final de la intervención.

Luego de los estudios basales se implementa el sistema HACCP la que incluyó las siguientes etapas:

1) Formación de un equipo HACCP con acto resolutivo (constituido por el Director del Hospital Regional de Ayacucho, el Jefe del Servicio de Nutrición y Dietética, el Jefe de la Unidad de Saneamiento Ambiental, el Jefe de Abastecimiento, el Jefe de la Unidad de Mantenimiento y el cocinero, cada uno de sus miembros con responsabilidades y funciones definidas.

2) Descripción y uso de la dieta proporcionada al paciente.

3) Elaboración y verificación del diagrama de flujo (todos los pasos seguidos desde la recepción de la materia prima hasta la distribución y consumo del alimento por el paciente).

4) Enumeración de todos los peligros de contaminación en cada fase del diagrama de flujo y elaboración del análisis de riesgos de estos peligros y estudio de las medidas preventivas para controlarlos.

5) Determinación de los puntos críticos de control (PCC), fase en la que puede aplicarse un control y que es esencial para evitar o eliminar un peligro para la inocuidad de los alimentos o para reducirlo a un nivel aceptable.

6) Establecimiento de límites críticos específicos y validados para cada PCC.

7) Se estableció un sistema de vigilancia visual para cada PCC que incluya las medidas correctoras necesarias, en caso que se detecte una pérdida de control en el PCC.

8) Establecimiento de procedimientos de verificación (análisis microbiológico de alimentos).

9) Establecimiento de un sistema eficaz y preciso de documentación y registro.

10) Evaluación continua del sistema HACCP para su mejoramiento.

Desde Marzo del 2002 se pusieron en funcionamiento los planes HACCP para comidas cocidas y comidas crudas (ensaladas) en el servicio de Nutrición. Se eligió los PCC de acuerdo al diagrama de flujo recomendado, instrumento diseñado según las referencia de DIGESA y de aplicación del sistema de Análisis de riesgos e Identificación y control de Puntos Críticos ${ }^{(4)}$. Se realizó la identificación de los PCC para comidas cocidas (almacenamiento de la materia prima, la cocción y la distribución) y comidas crudas (almacenamiento de la materia prima, desinfección y enjuague, distribución). Se establecieron los límites críticos para cada PCC, así como los responsables y metodología usada en el monitoreo, medidas correctivas, registro $y$ verificación de éstos.

Para determinar la eficacia del sistema se realizaron cinco estudios microbiológicos de los alimentos desde octubre del 2001 (basal) hasta diciembre del 2002 (final): para el análisis microbiológico de los alimentos proporcionados a los pacientes hospitalizados por el servicio de nutrición, se tomo como muestra al $20 \%$ de los pacientes hospitalizados en un día cualquiera; la cual considera un $95 \%$ de nivel de confianza (Epi Info v.6.0), se realizó un muestreo aleatorio estratificado por servicios y se tomó muestras de $100 \mathrm{gr}$. de alimento de las raciones proporcionadas a los pacientes.

Las muestras fueron procesadas en el Laboratorio de referencia regional con la técnica del número más probable, de acuerdo al protocolo de trabajo de análisis microbiológico de alimentos. Se definió alimento contaminado cuando se sobrepasaba del valor máximo de referencia microbiológica para comidas destinadas a pacientes en hospitales (Número Máximo de Microorganismos Permitidos - NMMP/g) ${ }^{(11)}$ Tabla 1.

Tabla 1. Valores de referencia microbiológicos para comidas destinadas a pacientes en hospitales.

\begin{tabular}{lc}
\hline Recuento de microrganismos & $\begin{array}{c}\text { Número máximo } \\
\text { permitido por } \\
\text { gramo (NMP/g) }\end{array}$ \\
\hline Mesófilos aerobios & $10^{4}$ \\
Coliformes totales & $10^{3}$ \\
Coliformes fecales & 10 \\
Salmonella spp & Ausencia \\
Staphylococo aureus enterotoxigénica & $10^{2}$ \\
Mohos y levaduras & $10^{2}$ \\
\hline
\end{tabular}

La vigilancia epidemiológica de las gastroenteritis intrahospitalarias se realizó en forma activa y selectiva; siguiendo la definición y metodología propuesta por la Dirección General de Epidemiología ${ }^{(12)}$, con reportes mensuales por servicio de hospitalización. Siendo excluidos como casos de gastroenteritis intrahospitalarias aquellas diarreas producidas por medicamentos, cambios en la dieta 
del paciente; durante la intervención se prohibieron el ingreso de alimentos de la calle.

Tabla 2. Resultados de la inspección Higiénico Sanitaria por ítem al inicio y final de intervención

\begin{tabular}{lccc}
\hline \multirow{2}{*}{ ITEM } & \multicolumn{3}{c}{ Puntaje } \\
\cline { 2 - 5 } & Ideal & Inicio & Final \\
\hline Edificación & 27 & 9 & 25 \\
Instalaciones sanitarias & 12 & 3 & 9 \\
$\begin{array}{l}\text { Abastecimiento de agua y } \\
\text { disposición de agua servida }\end{array}$ & 7 & 6 & 7 \\
$\begin{array}{l}\text { Almacenamiento de materia prima } \\
\text { y productos terminados }\end{array}$ & 6 & 6 & 6 \\
$\begin{array}{l}\text { Buenas prácticas de manufactura } \\
\text { Aspectos operativos }\end{array}$ & 36 & 26 & 33 \\
$\begin{array}{l}\text { Control de plagas y acceso de } \\
\text { animales }\end{array}$ & 3 & 3 & 3 \\
Total & 7 & 7 & 7 \\
\hline
\end{tabular}

Tabla 3. Incidencia mensual de Gastroenteritis Intrahospitalaria

\begin{tabular}{lccc}
\hline \multirow{2}{*}{ Meses } & \multicolumn{3}{c}{ Gastroenteritis Intrahospitalaria } \\
\cline { 2 - 4 } & Casos & $\begin{array}{c}\text { Pacientes } \\
\text { vigilados }\end{array}$ & Incidencia \\
\hline Noviembre & 28 & 238 & 11,7 \\
Diciembre & 30 & 208 & 14,4 \\
Enero & 35 & 268 & 13 \\
Febrero & 19 & 423 & 4,49 \\
Marzo & 13 & 200 & 6,5 \\
Abril & 21 & 561 & 3,7 \\
Mayo & 11 & 520 & 2,1 \\
Junio & 5 & 475 & 1 \\
Julio & 3 & 480 & 0,62 \\
Agosto & 0 & 537 & 0 \\
Setiembre & 0 & 666 & 0 \\
Octubre & 7 & 757 & 0,92 \\
Noviembre & 2 & 837 & 0,23 \\
\hline
\end{tabular}

La información fue ingresada a una base de datos previamente diseñada. Los resultados fueron expresados en frecuencias absolutas y relativas. Se realizó comparaciones entre la prevalencia basal y final tanto del análisis microbiológico de los alimentos como de la vigilancia epidemiológica de las gastroenteritis, estableciéndose riesgos relativos y considerando un valor $p<0,05$ como estadísticamente significativo. En el procesamiento y análisis de los datos se utilizó el paquete estadístico Epi Info. V.6.

\section{RESULTADOS}

En la línea de base se determinó que las condiciones higiénicas sanitarias del servicio fueron regulares, al final del estudio los resultados fueron muy buenos Tabla 2 .

El análisis microbiológicos de los alimentos al inicio del estudio reportaron contaminación en el 50\% (10/20) de los alimentos muestreados, al final de la intervención no se reportaron microorganismo alguno en las comidas servidas a los pacientes Figura 1.

En las gastroenteritis Intrahospitalarias el estudio inicial reporto una prevalencia de $11,7 \%$, en el transcurso de la implementación del sistema HACCP, las incidencias mensuales muestran una tendencia decreciente hasta $0,23 \%$. Tabla 3. Figura 2.

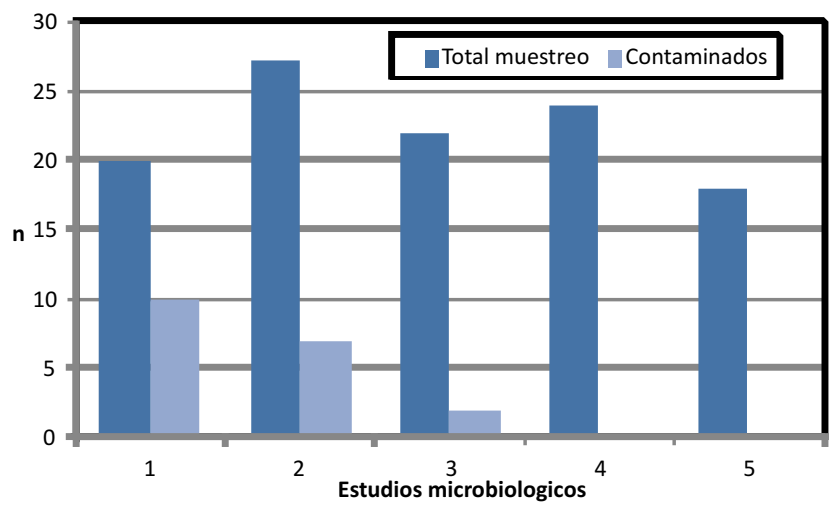

Figura 1. Contaminación microbiológica de los alimentos, durante la implementación del Sistema HACCP en el Hospital Regionl de Ayacucho 2001-2002.

La eficiencia del Sistema HACCP aplicado al Hospital para el control de la contaminación microbiológica de los alimentos se muestra en la evaluación al inicio de la intervención y los controles trimestrales de la cohorte de alimentos, los resultados microbiológicos de los alimentos al inicio y final de la intervención muestran un $\mathrm{RR}=0$ con límites de confianza exactos de 0,01<RR $<0,33$ al 95\% de confianza con un $\mathrm{p}$ valor 0,0005 , la que indica que los alimentos con sistema HACCP muestran una protección para la contaminación microbiológica de los alimentos. Las incidencias de Gastroenteritis Intrahospitalaria, al inicio y al final de la intervención, muestran un $R R=0.017$, con límites de confianza de cornfield de 0,01 < RR $<0,08$ al $95 \%$, con un valor de $p<0,05$, significativo para el sistema HACCP como factor de protección para las gastroenteritis intrahospitalarias. Tabla 4, 5 . 


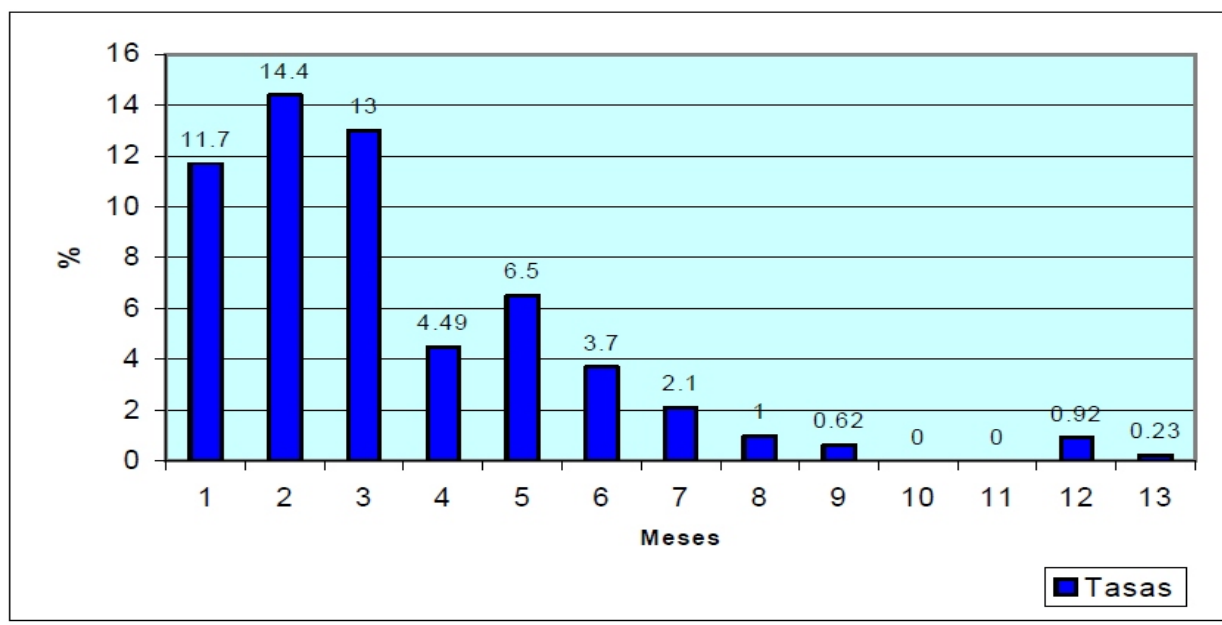

Figura 2. Incidencia de Gastroenteritis intrahospitalaria en servicios de hospitalización 2001 -2002

Tabla 4 Riesgo de Gastroenteritis Intrahospitalaria al inicio y al final de la implementación del Sistema HACCP.

\begin{tabular}{|c|c|c|c|c|}
\hline \multirow{2}{*}{ Variable Principal } & \multicolumn{2}{|c|}{ Implementación HACCP } & \multirow{2}{*}{$P^{*}$} & \multirow{2}{*}{$\mathrm{RR}^{*}(\mathrm{IC} 95 \%)$} \\
\hline & Inicio $(n=238)$ & Final $(n=837)$ & & \\
\hline $\begin{array}{l}\text { Gastroenteritis } \\
\text { Intrahospitalarias }\end{array}$ & $28(11,7 \%)$ & $2(0,23 \%)$ & $<0,05(0,000)$ & $0,02(0,00<R R<0,08)$ \\
\hline
\end{tabular}

* p y RR obtenidos al comparar el inicio y final de la intervención.

Tabla 5 Riesgo de contaminación alimentaria al inicio y al final de la implementación del Sistema HACCP.

\begin{tabular}{cccccc}
\hline \multirow{2}{*}{ Variable Principal } & \multicolumn{2}{c}{ Implementación HACCP } & \multirow{2}{*}{$\mathrm{P}^{*}$} & $\mathrm{RR}^{*}(\mathrm{IC} 95 \%)$ \\
\cline { 2 - 3 } & Inicio $(\mathrm{n}=20)$ & Final $(\mathrm{n}=18)$ & & \\
\cline { 1 - 3 } $\begin{array}{c}\text { Contaminación de } \\
\text { Alimentos }\end{array}$ & $10(50 \%)$ & $0(0 \%)$ & & $<0,05(0,004)$ & $0,00(0,00<\mathrm{RR}<0.33)$ \\
\hline
\end{tabular}

* $\mathrm{p}$ y RR obtenidos al comparar el inicio y final de la intervención

\section{DISCUSIÓN}

En la primera fase de la intervención (inspección higiénico sanitaria), se trato de medir los requerimientos sanitarios y procesos mínimos que se deben aplicar en todas las empresas que procesan alimentos, los resultados nos muestran un incremento en los puntajes de evaluación de los diferentes Items, de 60 puntos en la evaluación basal a 90 puntos al final de la intervención, esta situación se debe principalmente a la mejora en la edificación e instalación sanitaria del servicio de nutrición y dietética gracias al apoyo del proyecto GTZ, se muestra también un incremento en la evaluación de las buenas prácticas de manufactura la cual refleja los conocimientos y actitudes adoptadas por los trabajadores con respecto a una adecuada higiene personal y buenas prácticas en la manipulación de los alimentos, manejo de la limpieza y desinfección de los locales, producto de un trabajo constante en información y educación, en disposición adecuada de residuos y precauciones para evitar contaminación de los alimentos.

Existen estudios que recomiendan que los conocimientos sobre limpieza y desinfección son necesarios para garantizar la higiene de superficies que contactan con los alimentos, para aplicar los principios del sistema $(14,15)$, incrementar el cuidado de la manipulación (16), mejoramiento de las condiciones higiénicas sanitarias de las áreas de procesamiento de los productos alimenticios (17).

Sin embargo hay una preocupación por estandarizar científicamente un proceso de enseñanza-aprendizaje con metodologías educativas para aplicar el sistema HACCP, se 
han observado algunas barreras en el personal de nutrición como la falta de conocimientos, motivación, seguridad para aplicar el sistema y de parte de los directivos restricciones económicas similar a otros estudios en otro tipo de población (26), es importante hacer notar que el personal operativo del servicio es de educación limitada pero empoderado en aplicar los principios del sistema HACCP.

Durante la implementación del sistema HACCP se adecuó los siete principios del sistema a la situación hospitalaria del momento, esta forma se ha expresado y aplicado en otros estudios $(18,19)$, y se ha manifestado que es necesario poner en práctica completa el sistema HACCP en hospitales tal como lo hicimos en este trabajo.

Se han desarrollado los planes HACCP para alimentos crudos y cocidos en este hospital con la participación activa de los mismos trabajadores del servicio, esta forma de implementación ha surgido como propuesta del equipo luego del análisis de los diagramas de flujo de los diferentes comidas producidas en el servicio de nutrición y simplificando los planes para su fácil manejo, tal como fue sugerida en la experiencia de otros expertos con la finalidad de ser utilizadas realmente por los cocineros, ante la presencia de muchas recetas con diferencias de menor importancia. La mayoría de las propuestas de trabajo nos sugieren y plantean realizar planes HACCP para cada tipo o categorías de comidas como alimentos fríos, calientes, recalentados, alimentos abastecidos y otros $(19,20)$.

Para mostrar la eficiencia de nuestra intervención realizamos cinco análisis microbiológico de los alimentos durante la intervención (trimestrales), siendo los coliformes totales y fecales los más comúnmente aislados los mismos que fueron disminuidos y eliminados al final de la intervención estos resultados son coincidentes con los obtenidos en otros estudios $(21,22,23)$ y mostrándose como eficiente el sistema HACCP para contribuir al control de la contaminación de los alimentos en el cuarto y quinto control.

Se ha medido la eficiencia en base a los resultados de la tendencia de las incidencias de gastroenteritis intrahospitalarias, luego de la vigilancia epidemiológica activa y selectiva, los resultados mostrados nos señalan incidencias descendentes de $14,4 \%$ al inicio de la vigilancia a $4 \%$ y $0 \%$ a partir del sexto mes de intervención, estos resultados tienen relación con los resultados al segundo trimestre de los análisis microbiológico de los alimentos, y se ha podido observar una mayor incidencia de gastroenteritis intrahospitalaria con los servicios que tienen mayor estancia hospitalaria como traumatología, medicina y cirugía (tabla $\mathrm{N}^{\circ} 7$ ).

Hay pocos estudios relacionando al control de las gastroenteritis intrahospitalaria con los planes HACCP, si hay trabajos de identificación de puntos críticos y sugieren la implementación del sistema HACCP en hospitales, otras referencias microbiológicas hacen énfasis en que los principales microorganismos causantes de las gastroenteritis intrahospitalaria son producidos por virus en un $85 \%$ y bacterias en un $15 \%$, en nuestro trabajo no se realizó la confirmación microbiológica en los casos definidos como gastroenteritis intrahospitalarias.

Dentro de los factores de riesgo para las gastroenteritis intrahospitalarias pocos trabajos identifican a los servicios de nutrición y dietética como determinantes de importancia, en nuestro estudio hay una relación directa en intervenir preferentemente en el servicio de nutrición y dietética con el sistema HACCP y el control de las gastroenteritis intrahospitalarias.

La eficiencia del Sistema HACCP aplicado al Hospital para el control de la contaminación microbiológica de los alimentos se muestra en la evaluación al inicio de la intervención y los controles trimestrales de la cohorte de alimentos, los resultados microbiológicos de los alimentos al inicio y final de la intervención muestran un $\mathrm{RR}=0$ con límites de confianza exactos de 0,01<RR < 0,33 al 95\% de confianza con un $p$ valor 0,0005 , la que indica que los alimentos con sistema HACCP muestran una protección para la contaminación microbiológica de los alimentos. Las incidencias de Gastroenteritis Intrahospitalaria Tabla 8, al inicio y al final de la intervención, muestran un $\mathrm{RR}=0.017$, con límites de confianza de cornfield de 0,01<RR $<0,08$ al $95 \%$, con un valor de $p<0,05$, significativo para el sistema HACCP como factor de protección para las gastroenteritis intrahospitalarias.

Nuestra experiencia puede ser usada para que otros hospitales evalúen la implementación de este sistema, a fin de garantizar el consumo de alimentos inocuos para los pacientes y personal, así como controlar las enfermedades producidas por alimentos en los hospitales.

\section{Correspondencia:}

Gualberto Segovia Meza.

Correo electrónico: guaber2@yahoo.es

Teléfono: 995229575

\section{REFERENCIAS BIBLIOGRÁFICAS}

1. Arrospide L. Implementación del Sistema de Análisis de Riesgos y Puntos Críticos de Control - HACCP. Facultad de Ingeniería Industrial. Universidad de Lima. 43 pp. 1998, Lima. Perú.

2. Icochea (1 994). "Una muy Buena Decisión - HACCP". Expopesca. Año 2, № 12. Chile.

3. DGCSBS (1993). "Manual de Aplicación del Análisis de Riesgos, Identificación y Control de Puntos Críticos". 
Dirección General de Control Sanitario de Bienes y Servicios. México D.F., 48 pp.

4. Ducar P. (1991). "El Sistema de Análisis de Riesgos y Puntos Críticos. Su Aplicación en la Industria de Alimentos". Edit. ACRIBIA. Zaragoza. España. 327 pp.

5. Romero J. (1996). "El Sistema de Análisis de Peligros y Puntos Críticos de Control, aplicado paso a paso al aseguramiento de la calidad de productos alimenticios", $1^{\circ}$ edición. Edit. Corporación Colombiana Internacional. Colombia. 119 pp.

6. CCl (1 993). "Sistemas de Gestión de la Calidad, ISO 9000: Directrices para empresas de países en desarrollo". Centro de Convenio Internacional/UNCTAD /GATT. Ginebra. Suiza. 228 pp.

7. MOSSEL, D. (1985). "Microbiología de Alimentos Fundamentos ecológicos para garantizar y comprobar la inocuidad y la calidad de los alimentos". 1 Edición. Edit. ACRIBIA S.A Zaragoza, España. 373 pp.

8. MINSA (1979). "Norma y Procedimientos Sanitarios para el Abastecimiento, Transporte, Conservación, Preparación y Servido de Alimentos en Hospitales y Servicio de Alimentación del Ministerio de Salud. Resolución Directoral No 0047-79 - SA/DS. Ministerio de Salud. Lima. Perú.

9. MINSA (1999). "Administración de Residuos Hospitalarios - Infecciones Intrahospitalarias". Ministerio de Salud. Lima. Perú.

10. Reglamento sobre vigilancia y control sanitario de alimentos y bebidas DS-007/98.S.A.

11. Mossel D. Microbiología de alimentos - Fundamentos ecológicos para garantizar y comprobar la inocuidad y la calidad de los alimentos. 1 era. Edición. Editorial Acribia S.A. Zargoza. España. 1985. 373 pp. Pag 195.

12. OGE - Ministerio de Salud. Protocolo: Estudio de Prevalencia de Infecciones Intrahospitalarias. OGE. Lima - Perú. 2000.

13. DIGESA (2002), Guía de Autocontrol Sanitario basado en el Sistema HACCP para servicios de alimentación y nutrición de Hospitales y Clínicas. Lima - Perú, 2002.

14. Setiabuhdi $M$, Theis $M$, Norback J. Integrating hazard analysis and critical control point (HACCP) and sanitation for verifiable food safety. J Am Diet Assoc 1997;97(8):889-91.
15. Salvat G, Colin P. Cleaning and disenfection practice in the meat industries of Europe. Rev Sci Tech 1995;14(2):313-41.

16. Little $\mathrm{CL}$, Louvois $\mathrm{J}$ de. The microbiological examination of butchery products and butchers'premises in the United Kingdom. J Appl Microbiol 1998;85(1):177-86.

17. Shanaghy N, Murphy F, Kennedy K. Improvements in the microbiological quality of food samples from a hospital cook-chill system since the introduction of HACCP. J Hosp Infect 1993;23(4):305-14.

18. Ángel Caballero Torres, Maiviv Carreño Ramírez, Thaimi Cárdenas Valdés, Ondina Grave de Peralta y Fidel Peraza Escoto proceso de enseñanzaaprendizaje y utilización del sistema de análisis de peligros y puntos críticos de control en alimentos. Revista Cubana Aliment Nutr 2002;16(1):35-41.

19. Hiroshi Yoshikura, Workflow from Clean to Dirty, HACCP and Inclusiveness Principles in Effective Implementation of Hospital Infection Control. Jpn. J. Infect. Dis., 53,124-125, 2000

20. Italo F. Angelillo,DDS, Mph y Col. HACCP e higiene alimenticia en hospitales: Conocimientos, actitudes y prácticas del personal de los alimentos - servicios en Calabria, Italia. Intectión Control and Hospital Epidemiology, 22(6), junio del 2001.

21. Oliveira $M R$, Batista $C R$, Aidoo KE. Application of Hazard Analysis Critical Control Points system to enteral tube feeding in hospital J Hum Nutr Diet. 2001 Oct;14(5):397-403.

22. Bobeng BJ, David BD. HACCP models for quality control of entree production in hospital foodservice systems. II. Quality assessment of beef loaves utilizing HACCP models. : J Am Diet Assoc. 1978 Nov;73(5):530-5.

23. Wilkinson PJ, Dart SP, Hadlington CJ.Cook-chill, cookfreeze, cook-hold, sous vide: risks for hospital patients? J Hosp Infect. 1991 Jun;18 Suppl A:222-9.

Recibido: 14/01/2012

Aceptado para publicación: 24/02/2012 